\title{
Resilient urban form to pandemics: Lessons from COVID-19
}

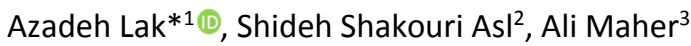 \\ Received: 10 May 2020 \\ Published: 30 Jun 2020
}

\section{Abstract}

Background: The worldwide emergence of future pandemics emphasizes the need to assess the pandemic resilient urban form to prevent infectious disease transmission during this epidemic. According to the lessons of the COVID-19 outbreak, this study aimed to review the current strategies of responding to pandemics through disaster risk management (DRM) to develop a pandemic-resilient urban form in phases of response, mitigation, and preparedness.

Methods: The research method is developed through desk study was used to explore the current literature of urban form responded to COVID-19 pandemic and for the text analysis; qualitative content analysis was applied developing a conceptual framework.

Results: To create pandemic resilient urban form, this study proposes principles to enhance the urban form resiliency in 3 scales of housing, neighborhoods/public spaces, and cities. These principles focus on the concept of resilient urban form from new perspectives focusing on the physical and nonphysical aspects of resilient urban form, which develops a new understanding of pandemics as a disaster and health-related emergency risks. The physical aspect of resiliency to epidemic outbreaks includes urban form, access, infrastructure, land use, and natural environment factors. Moreover, the nonphysical aspect can be defined by the sociocultural, economic, and political (including good governance) factors. By providing and enhancing the physical and nonphysical prerequisites, several benefits can be gained and the effectiveness of all response, mitigation, and preparedness activities can be supported.

Conclusion: As the pandemic's disruptions influence the citizens' lifestyle dramatically, the prominent role of place characteristics in the outbreak of pandemics, policymakers, urban planners, and urban designers should be pulled together to make urban areas more resilient places for epidemics and pandemics.

Keywords: Pandemic resilient urban form, Disaster risk management (DRM), COVID-19, Urban planning and design

Conflicts of Interest: None declared

Funding: None

\section{*This work has been published under CC BY-NC-SA 1.0 license. \\ Copyright $\odot$ Iran University of Medical Sciences}

Cite this article as: Lak A, Shakouri Asl Sh, Maher A. Resilient urban form to pandemics: Lessons from COVID-19. Med J Islam Repub Iran. 2020 (30 Jun);34:71. https://doi.org/10.47176/mjiri.34.71

\section{Introduction}

Over the past decades, more and more studies have been performed on the relationships between planning, design, and promoting health and well-being in communities that mainly focus on chronic diseases, hazards, disasters, and vulnerable groups.

Corresponding author:Dr Azadeh Lak, A_Lak@sbu.ac.ir

1. Faculty of Architecture and Urban Planning, Shahid Beheshti University, Tehran, Iran

2. Science and Research Branch, Islamic Azad University, Tehran, Iran

3. School of Management and Medical Education, Shahid Beheshti University of Medical Sciences, Tehran, Iran
These studies aim to empower the cities with more resilience and ability to overcome crises and hazards and provide more sustainable and inclusive human settlements. However, not enough research has focused on infectious diseases and pandemics from the perspective of urban plan-

$\uparrow$ What is "already known" in this topic:

Despite the significant impacts of transmission of infectious diseases in cities, limited research exists on how urban form can enhance resilience by increasing the abilities to plan for, absorb, recover from, and adapt to adverse outbreaks due to global experiences of COVID-19.

\section{$\rightarrow$ What this article adds:}

This study aimed to develop a framework for pandemic resilient urban form according to response, mitigation, and preparedness phases of DRM in 3 spatial scales of housing, neighborhood/ urban spaces, and city. 
ning and design. Historically, several epidemics or pandemics had emerged such a s Athens, Antoninus, Cyprus, Justinianus, Swine flu, Cholera, Ebola, and Smallpox, which led to catastrophes, widespread illnesses, deaths, and also caused long-lasting and irrecoverable damages on different countries' environments, societies, economies, and even individuals' mental health (1).

The COVID-19 outbreak as a widespread phenomenon has adversely influenced several countries worldwide, regardless of their income and political commitment to improve health and wellbeing as an essential item of the Sustainable Development Goals (SDGs) agenda (2).

The current pandemic has attached far too much importance to finding strategies for making cities more resilient to outbreaks, as cities are on the frontline of deadly epidemics, other globalized crises, and further damages and drawbacks. Pandemic-resilient cities can be achieved through creativity and taking advantage of cutting-edge technologies and improvements in science, medicine, ethics, legal systems, and sociopolitical systems (2). Also, urban planning and design play a prominent role in building resilience.

Based on recent studies, high density of population, frequent and complex interactions, shortage of health care facilities in cities (2), and environmental pollution (3) are among the elements that exacerbate pandemic situations and raise difficulties in mitigation, prevention, and control of outbreaks, particularly in developing countries.

Cities especially as dense centers of the mobile population, intensified land uses, transportation services, and social interactions are more prone to pandemics than rural areas. Intercity and intra-city transporting systems, airports, ports, hubs, CBDs, and other centers of dense transactions pose more threats to urban areas and make them more vulnerable to pandemics. Accordingly, to assume the control of the pandemics, cutting down the interactions between the citizens and minimizing the connections and relationships between urban areas and citizens are of the most challenging strategies (4). On the one hand, the essence of reducing social interactions, face to face connections, and outdoor activities for declining the speed of virus spread is undeniable; on the other, it is somehow against the entity and characteristics of modern cities and will certainly unfavorably impact urban life as well as the form, activity, and meaning of urban areas. In 2015, Sendai Framework for Disaster Risk Reduction 2015-2030 (5) and the Global Assessment Report 2015 (6) proposed a new approach to operationalize a resilient holistic approach for DRM by focusing on the resilience of development goals and human health and well-being. The resilience for the disaster risk management (DRM) concept has been widely developed during the last decade, creating a new ecological resilience approach (7) that requires new and effective strategies for preparedness, response, and mitigation $(8,9)$. According to Bell et al (10), new approaches for preparedness, mitigation, and response are claimed as essential strategies that may not be easily scalable upward to address huge, densely populated areas, especially in developing countries. Rivera and Sillah (11) defined the phases of preparedness, mitigation, and response as below:
"Preparedness level (phase) aims to increase the preparedness and response capabilities of cities against outbreaks. Mitigation (Prevention) is known as the structural/nonstructural measures and activities undertaken to limit the adverse impact of epidemics. Responses are defined as the provision of emergency services and public assistance during or immediately after pandemics to reduce health impacts by controlling disease transmission as well as ensuring public safety and meeting the basic subsistence needs of the affected people." (12).

This study aimed to develop a conceptual framework for the urban form resilient to pandemics by adopting resilience from the DRM approach in urban planning and design. The results of this paper can be used to offer possible suggestions and recommendations in 3 levels of housing, neighborhood/ public places, and city to enhance response, mitigation, and preparedness phases against epidemics in the future. This new approach is in line with Carmona et al (2014) structured "resilient urban planning and design" around dimensions (morphological, perceptual, social, visual, functional, and temporal) and processes (development, control, and communication) for considering a resilient form to outbreaks (13).

\section{Development of a conceptual framework: Ur-}

\section{ban form resilient to pandemics}

Resilience aims to remove the problematic issues of uncertainty $(13,14)$ through the vulnerability analysis matrix, urban governance, prevention, and uncertainty-oriented planning and urban form (15). Looking at the trend in resilience to the pandemic, it can be defined as the ability of urban environments to absorb a blow without a meaningful change in structure or function (2). The main benefit of using the term resilience for DRM, as a paradigm that assists in developing disaster risk management strategies, is conceptual/theoretical and methodological advancement (15). Accordingly, developing a conceptual framework for the resilient urban form is based on the response, mitigation, and preparedness phases in 3-fold scales of housing, neighborhood/public places, and within cities (Fig. 1).

Throughout history, scholars found solutions during some pandemics and in several other outbreaks they could only offer guidelines for managing infectious diseases to minimize the drawbacks and losses. The guidelines were only effective when combined with citizens' participation and cooperation. In other words, as the pandemic spreads, citizens should follow the guidelines and instructions to slow down the virus spread on different levels (1). These guidelines are set to ensure people's health and well-being through reducing poverty, providing amenities, services and networking patterns, to provide training for necessary behavioral changes, and to enhance social relations, values, and commitments. To develop a conceptual framework, 3 phases of DRM were identified as different aspects of urban resilience to pandemics. For each of the domains of DRM, the conceptual framework has been developed and detailed to define the relevant indicators and criteria.

The outlined conceptual framework identifies physical and nonphysical dimensions of built environments (13) to 


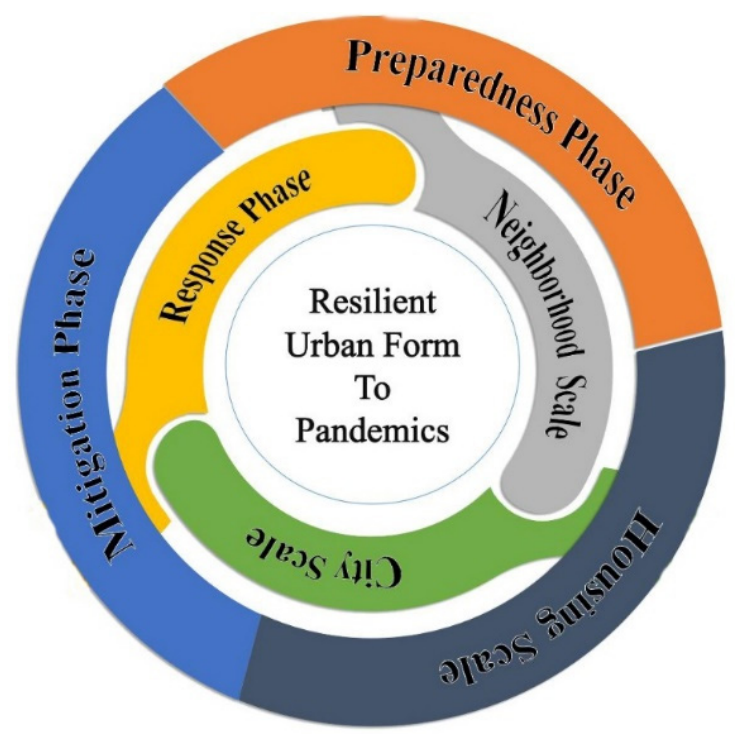

Fig. 1. The proposed framework for resilient urban form to pandemics

face pandemics using a social and environmental determinant of health perspective. Associations drawn from previous studies were used to guide the development of the proposed conceptual framework. In turn, the conceptual framework was used to identify spatial measures of resilient urban form to epidemics in 2 dimensions of physical and nonphysical that may be associated with selected 3 scales in cities (Fig. 1).

The development of the conceptual framework is according to the $3 * 3$ matrix of DRM phases and urban form spatial scales. DRM phases include mitigation, response, and preparedness phases which need to be accountable in these spatial scales (housing, neighborhood, and city) in time of a pandemic. According to the dynamic nature of a resilient city and flexibility to changes during an outbreak of contagious diseases, the static $3^{*} 3$ matrix is turned into $2 \mathrm{dy}$ namic circular interrelated diagrams which can be applied in different situations, depending on the time and place of the pandemic outbreak, which could lead to phases in different spatial scale.

\subsection{Responses phase}

To ensure an effective response to a pandemic, both hardware (health care, health infrastructure, etc.) and software (developed and tested protocols, proper knowledge, qualified medical team and staff, etc.) of prevention-functioning surveillance systems must be provided. To prompt an effective response to pandemics, the different aspects of urban life needs to change. Thus, the response phase must be based on the early adaptation of a pandemic preparedness plan that would be developed for a virus originating abroad. The response phase includes various actions, such as response coordination, surveillance, and monitoring of illness trends, disease containment and mitigation, delivery of countermeasures, and public communication (10).

Promoting personal and environmental hygiene in the epidemic situation, providing sufficient shared spaces indoors for quarantine and lockdown for large urban families, providing spaces in the residential area for providing care to ill people, subsiding for suitable ventilation to maintain optimal humidity (range of 40 to $60 \%$,) and providing filtering indoor air for ill families can be illustrated in housing scales.

Based on the importance of health at the neighborhood level in pandemics (16), the physical dimension of response plans in neighborhoods may include the following activities: (1) enhancing infrastructures for more effective use of cell phones, smart apps, and the internet in the monitoring and surveillance of illness trends by community centers at neighborhood scales; (2) preventing and controlling communal trips in neighborhoods; (3) asking for public participation for public control at neighborhood level; (4) instructing local people to enforce local trips restrictions at neighborhood level; (4) controlling the use of parks, community gardens, and playgrounds according to social distancing protocols of local authorities and city councils; (5) promoting biking and walking while considering social distancing in neighborhoods; (6) asking local people to control population density in neighborhood centers; (9) decontaminating and sanitizing public places and urban furniture in neighborhoods frequently; (10) placing homeless people in vacant buildings such as schools and churches; (11) restricting large public gatherings, canceling programs, and events, and closing indoor recreational facilities; (12) providing the facilities of teleworking and pay in neighborhoods; (13) closing theatres and concert venues, museums, restaurants, amusement parks, and other places of leisure; (14) establishing an epidemic prevention area based on (EPA) public health spatial unit in neighborhoods; (15) providing a well-developed, reliable, and transparent informative system to increase public awareness; (16) developing a well-organized, appropriate and place-based population documentary and database for rapid delivery of countermeasures in neighborhoods; (17) and providing several local mutual aid groups to support vulnerable groups during isolation.

The functional-spatial structure of the city can be improved by taking the following essential measures: (1) supporting urban and local services of medical facilities in different scales; (2) identifying the right locations for EPA- 
based public health in cities; (3) controlling airports, arrivals, and departures of passengers to the city; (4) reducing the number of daily travels, and as a result reducing air pollution and traffic; (5) mobilizing the response coordination system with nongovernmental institutions, community, and faith-based organizations (NGOs) at the city scale; (6) promoting mobilization integrated risk management; (7) enhancing infrastructures for more effective use of cell phones, smart apps, and the internet for monitoring and surveillance of illness trends at the city scale; (8) and enhancing public knowledge about epidemics. Moreover, establishing the database of transportation, land, and infrastructure planning information systems, such as websites or phone hotlines that provide information about current levels of traffic congestion may be useful to individuals and official responders in these situations $(4,17,18)$.

Recent studies indicate that cutting-edge and diverse technologies can support the preparation of more comprehensive and accurate health and hygiene databases, which can be helpful during outbreaks in making optimum decisions and minimizing the risk. Some monitoring technologies provide the chance to ensure whether all people with symptoms are recognized and quarantined (4).

\subsection{Mitigation phase}

This phase aims to mitigate the urban issues in pandemics; for instance, densely populated areas, increased connectivity, poor quality of sanitation ventilation, and insufficient water supply are known as important factors that increase outbreak risks. Dealing with dense issues, harnessing novel data streams, and new technological advances to manage epidemics, mitigating false information on social networks, and creating informal technical networks are matters of overriding concern in the preparedness stage (17, 18). Accordingly, in the resilient urban form, the mitigation phase can be considered from 2 different aspects: the physical and nonphysical dimensions $(13,19)$.

\subsubsection{Physical dimension}

Different characteristics of a city can play a prominent role in mitigation and adaptation to pandemics. Some of the most significant of such characteristics in physical dimensions can be classified as urban form (built environment), access (transportation), and infrastructure (transportation), land use, and natural environment (20).

\section{- Built Environment Attributes}

Urban form determines the densities and relative locations of housing, jobs, and services in a city. Consequently, it influences the transporting choices of the population. Accordingly, the average of plot sizes, the height of buildings, the ratio of mass and space, density, and clustering are elements of great influence on disease spread (16). The high density of population, poor housing, and overcrowding can also contribute to vector proliferation (21). However, compacted urban form and higher network density can lead to insufficient social distancing in larger or typically more connected cities, which accelerates the growth of disease transmission and outbreaks. It can also end up in the crea- tion of reservoirs for infectious diseases in the future. Moving towards self-sufficiency and resource-efficient centralized neighborhoods, providing the sufficient ratio of open spaces in neighborhoods, taking territorial theory into account in design processes (by providing semi-public and semi-private or shared open spaces in residential buildings for planting, playing and working out in pandemic situations), preventing the formation of crowds in neighborhoods and cities can make neighborhoods more resilient to pandemics.

To reinforce the urban form for facing and dealing with pandemic diseases at the city scale, a new approach towards the concepts of population and density should be adopted to shorten the risk of virus spread (for instance, a threshold level of population density of 175 people per square mile for influenza pandemics) (22). Increased open spaces in neighborhoods will enable people to socialize without being packed (social distancing). EPA planning and design as the substitution of neighborhood centers or HUB/CBD can also protect and strengthen functional-spatial structure of cities during pandemics. Another important factor is the effective distance between cities. Accordingly, the smaller the effective distance, the more convenient the connection between the 2 cities, the larger the scale of the population flow, the easier the spread of an epidemic across regions (23). Thus, cross-regional cooperation is vital to control and reduce epidemic spreads cross regions as a result of population flow between cities.

In housing scale, improving the quality of housing (Poor housing can lead to the proliferation of insect and rodent vector diseases and geo helminthiases.), facilitating the filtration, ventilation and sanitation system of buildings and homes, designing semi-open spaces in housing design like balconies for planting and pleasure, designing shared sanitation-sanitation facilities shared by multiple householdsand providing sufficient public open spaces in the residential settlement can foster resilient housing in epidemics as well as prevent the proliferation of insect and rodent vector diseases, geo-helminthiases, and zoonotic diseases in residential settlements (21).

\section{- Access (Transportation) and infrastructure}

The structure of the urban movement network and the hierarchy of roads have a significant impact on human mobility as a key element in the spread of the epidemic diseases. Accordingly, proper quantification of human mobility in a city is substantial for assessing the large-scale spatial transmission of infectious diseases and improving epidemic control (24). Inefficient network design and poor quality of network can cause heavy traffic, which acts as an obstacle in having quick access to health centers for patients, doctors, or hospitals' staff. Crowded public transit vehicles can also play a prominent role in increasing the rate of viruses spread (25).

Designing flexible bike-friendly and walkable neighborhoods and cities (with estimating safe distancing); providing wide sidewalks and bike paths, entryways, and public transit stations; designing transit neighborhood development (TND) that supports uncrowded public transportation with social distancing and density help to make public 
transportation and movement safer. Moreover, policies such as decreasing heavy traffics, which is an obstacle in emergencies; planning for well-facilitated roads and streets, and changing passengers' behaviors via signs and floor markings to show that safe distances between riders and passengers can be applied as an effective intervention in movement systems to increase the pandemic resiliency at neighborhood and city levels are of high importance.

Moving towards smart cities allows policymakers to make informed and effectual decisions and sound policies by which urban governance can be improved $(17,18)$. As a matter of fact, it is of vital importance to develop prerequisite infrastructures to make our cities smart. The absence of calibration between smart city technology suppliers can lead and is leading to no communication between cities and data platforms and result in a nonproductive system in the case of virus epidemics (26). Thus, there is an essence to democratize the smart city technology sphere, which promotes equity, trust, and transparency between stakeholders as an important prerequisite of beneficial and effective cooperation in dealing with disease outbreaks (26).

\section{- Land use}

Fortifying cities for dealing with pandemic situations focusing on land use system can be considered from 2 main areas:

1. Enhancing the quality and quantity of the health and medical services as a multilayer system (All parts of the city should be under service of health services with different scales of the city, region, and neighborhood.)

2. Other activities and services should provide supportive amenities for health and medical facilities and services

Elements that can determine whether a city can effectively manage a pandemic are as follow: (1) promoting equal well-being for all citizens and reducing inequity in slums; (2) planning and designing decentralized amenities within neighborhoods and city centers; (3) a well-distributed public open spaces; (4) distributing retails within neighborhood instead of concentrating them in the center; (5) classifying land use according to the health status in pandemics; and (6) ensuring quality and accessibility of hospitals, internal care units, hospital beds, and respirators $(4,27)$.

Designing the spatial structure of the city in line with EPA integrated health and hygiene system can help decline disease transmission and epidemics. Providing adequate quality and quantity of public open spaces as contexts and beds of social interaction is also of paramount significance. Large parks allow citizens to engage in outdoor physical activities in lockdown situations and to practice social distancing. This can help reduce the further mental and physical drawbacks of not going outdoors or cutting down social interactions, such as loneliness and depression (28).

\section{- Natural environment}

A recent study found that epidemics like COVID-19 may be transmitted through 2 mechanisms: air pollution-to-human transmission and human-to-human transmission. The combination of air pollution and meteorological conditions (with high moisture $\%$, low wind speed, and fog) accelerates epidemic diffusion in addition to further drawbacks on the population's health, economy, and society (29). Thus, air pollution can be relatively considered as a more critical issue, while the outbreak of pandemics and the essence of minimizing urban pollution by controlling urban development, population growth, industrialization, and deforestation is undeniable (29).

According to Beelen et al (2014), the need to draw attention to the continuing effects of air pollution on health is necessary. In this regard, decreasing the high concentration of nitrogen dioxide, a noxious gas, particulate air pollutants emitted by motor vehicles, power plants, and industrial facilities are a platform to support the viral diffusion infectivity (31), increase hospitalizations for respiratory virus bronchiolitis (32), increase asthma incidence (32) and damage the immune system (33). Overall, the deindustrialization of polluting industries and moving toward sustainability should be considered as a need in urban developments as stepping stones for climate change mitigation improvement of the environment, atmosphere, air quality, and especially health of populations (29).

\subsubsection{Nonphysical dimension}

The recent worldwide COVID-19 epidemic has demonstrated, albeit, on a much larger stage, the lessons learned from past outbreaks that resilience in sociocultural, economic, and governance is an essential and cost-effective feature of a health system addressing complex challenges. According to a study (12), "Resilience is fostered not just by science-based decisions and coordination, but also via redundancy to ensure buffer capacity when a particular system collapses."

The inequity of resources-especially in urban shantytowns- poor quality, and deteriorated urban areas are potentially more vulnerable, as there are usually more crowded areas with fewer open spaces and poor quality roads and networks. Most of the time, these areas suffer from low environmental hygiene levels. Homeless people, people in inadequate, unhealthy crowded houses, or those in shared, dirty homes and even tents are more exposed to the risk of diseases (4). Inequity to access to education, resources, and quality of life, controlling ethnic enclaves for health and sanitary lifestyle, and declining social capital, and social networks in the new development may relatively emphasize the importance of social resilience in overwhelming pandemics (34). In other words, reducing poor urban areas and eradicating socioeconomic inequities to enhance economic resilience can be considered as perquisites of inclusively local and self-sufficient economy (4).

Governance or political resilience can be considered to provide health infrastructure as well as health management for better management of pandemics (4). Resiliency in governance can be defined as the sharp action in adopting a combination of proactive surveillance, routine communication, rapid isolation, and personal and community protection (eg, social distancing) measures and deciding to act fast and efficiently to global surveillance and alert systems to reduce the impact (34). Moreover, adaptation, pre-exist- 
ing pandemic plan, and planning process may facilitate intersectoral work, decision making, and rapid development of a public communications campaign. Reinforcing the decision making and the planning process by taking advantage of decision support and information systems, such as Decision Support Systems (DSSs), Planning Support Systems (PSSs), and Geographic Information Systems (GIS), along with setting up urban dashboard, can help governance to decide more precisely in pandemics (4). Providing sanitary control for areas approximated to open public spaces and subway lines for zoonotic infectious diseases and trying to decrease inequity in slums in mega cities are supposed to be effective measures to prevent pandemics.

A smart city can guarantee more efficient interferes during pandemic times. According to Inn, smart cities can contribute to better management of pandemics in the following ways: providing the opportunity to identify, track, and forecast outbreaks through big data analytics; improving public security through enhanced facial recognition and infrared technologies; using drones or smart robots to sterilize, deliver supplies, and assist surveillance; assisting research and studies on viruses and relevant issues as well as potential treatments, including smart apps and Al-powered chatbots, to support virtual communication at times of restrictions face to face interactions (1).

For instance, to design a smart city development in China, the government used artificial intelligence (AI), including surveillance cameras, drones, facial recognition technology, large data collection and analysis, tracking apps, and QR codes to link travel history and medical data. Besides, China has used various technologies to fight COVID-19 and optimize its surveillance system to enhance public security and the livelihood of its people. For instance, the combination of infrared cameras and the facial recognition system is able to detect any individual with high temperature and whether the person is wearing a mask. At the same time, China has developed robots equipped with AI to reduce the potential risk of exposing the health authorities to COVID-19, while conducting manual measurement of body temperature (26).

\subsection{Preparedness phase}

The preparedness phase emphasizes the increase in the capacities for dealing with outbreaks. Some cities with prior experience of huge disease outbreaks have already developed purpose-built surveillance systems using digital and web-based information (35). Some other local authorities have established surge capacity and contingency plans, including strong links with health care facilities and national coordination bodies (36). "In some urban settings, complex simulation exercises have been run, which allow modeling disease spread and determining the optimum public health actions to control the diseases spread" (37). Such simulation systems are both very efficient assessment and evaluation tailored for urban settings and can be useful for preparedness planning and design.

All the developed strategies and innovating technologies in controlling the pandemics try to minimize the person-toperson interaction. The controversial issue is how to main- tain livelihoods, social life, and mental health while minimizing face to face interactions. This may be fulfilled through approaches that make the most of emerging virtual places (created online in cyberspace through internet technologies) as well as the real time data to create context-appropriate suppression strategies at local, regional, national, and global levels (38). Cutting-edge technologies and virtual socializing platforms can play a prominent role in dealing with this complex issue. To sum up, at the time of infectious disease outbreaks, such as COVID 19, adopting appropriate measures, including social distancing, isolation, quarantine, is vital (39). While citizens should accept the posed limitations and follow the restrictions and measures, planners can play a key role in predicting and estimating people's requirements for lockdown situations.

\section{Conclusion}

The outbreaks of infectious diseases have resulted in wide-ranging socioeconomic and political consequences, including loss of lives and disruption to the daily life of residents. Furthermore, emerging epidemics originate in urban settlements. Therefore, urban planning and design must put much effort into increasing the cities' resiliency to cope with pandemics through DRM and to consider resilience as a system's ability to accommodate variables and unexpected conditions without catastrophic failures and changes.

Many communities have aimed to increase the resilience of infrastructure and public services as well as the resilience to economic shocks and disasters (4). Therefore, the resilient urban form is claimed as a new approach to incorporate resilience and flexibility. Thus, it must encompass resilient physical aspects of cities as well as nonphysical aspects by enhancing socioeconomic and political (good governance) resilience. Accordingly, preparedness for pandemics can be implemented through taking the following measures: improving the quality of life of people living in slums; creating flexible and alternative public transport; and planning for population density and connectivity, mixed-use development, and infrastructure redundancy (e.g., ensuring the availability of multiple access routes, diverse transport systems, multiple water and energy sources, and emergency supplies for communities). Also, implementing innovative technologies and "smart cities" policies in housing, neighborhood, and city levels can be helpful to monitor disease transmission in the response phase.

Socioeconomic resiliency can be improved by taking the following actions: increasing social networking (which can indirectly help in emergency leadership); enhancing diversified economic structure to maintain employment and business activity despite economic shocks; and providing public and health services, insurance coverage, employment, and income. Moreover, decreasing deprived urban areas and eradicating people's economic insecurity are also vital. The current crisis has revealed that many people do not have the financial resilience to deal with an unexpected hardship imposed upon them. A resilient society in future must eradicate the individual's economic insecurity that is mainly due to the lack of appropriate legislation to protect the workforce, lack of comprehensive social security and 
welfare systems to mainly cover the lower income groups, and the no income groups of each society. Importantly, good governance and political resilience are claimed as the core of measures to achieve effective leadership and coordination in DRM through local leadership in planning and implementation, providing public health network and EPA on the function-spatial structure to deal with epidemics, and providing sources for financing.

In COVID-19 pandemic, mew concepts such as social distancing and virtual places have emerged as a new phenomenon. Accordingly, training and educating people can be applied as the root of pandemic resilient cities to enhance them for active public participation in new urban virtual spaces, heath and sanitary behaviors, and engaging local leadership. Ensuring better preparedness and response for epidemics in urban areas will require a substantial consideration to strengthen various capacities through the resilient urban form. However, urban planning and design must define a new concept of place (emphasized on the social aspect of place). The emerging new concept of place causes changes the way people live, work, and interact in resilient built environments. This new conception is essential for policymakers and urban planners and designers to strengthen systems and local capacities to prevent the spread of infectious diseases in the future.

The suggestions for future studies focus on defining qualitative studies based on the pandemic resilient city features through the consensus of experts in the fields of urban health, urban resilience, and urban planning and design. Also, quantitative and field studies could lead to the exploration of the association between the features of resilient cities that have experienced the COVID-19 pandemic and disease transmission.

\section{Conflict of Interests}

The authors declare that they have no competing interests.

\section{References}

1. Omidi M, Maher A, Etesaminia S. Lessons to be learned from the prevalence of Covid-19 in Iran. Med J Islam Repub Iran. 2020;34(1):398-9.

2 Zhou C, Su F, Pei T, Zhang A, Du Y, Luo B, et al. COVID-19: challenges to GIS with big data. Geogr Sustain. 2020;1(1):77-87.

3. Reyes R, Ahn R, Thurber K, Burke TF. Urbanization and infectious diseases: general principles, historical perspectives, and contemporary challenges. In: Challenges in Infectious Diseases. Springer; 2013. p. 123-46.

4. Mollalo A, Vahedi B, Rivera KM. GIS-based spatial modeling of COVID-19 incidence rate in the continental United States. Sci. Total Environ. 2020;728(6):13884

5. UNISDR U. Sendai Framework for Disaster Risk Reduction 2015 2030. 2015. United Nations Int Strateg Disaster Reduct Geneva.

6. UNISDR. Global Assessment Report on Disaster Risk Reduction 2015: Making Development Sustainable: the Future of Disaster Risk Management. UN; 2015.

7. Wallace D, Wallace R. Urban systems during disasters: factors for resilience. Ecol Soc. 2008;13(1).

8. Lawton M, Nahemow L. Ecology and the aging process. 1973 [cited 2019 Jan 14]; Available from: http://psycnet.apa.org/record/200415428-020.

9. Kinsman J, Angrén J, Elgh F, Furberg M, Mosquera PA, Otero-Garcia $\mathrm{L}$, et al. Preparedness and response against diseases with epidemic potential in the European Union: a qualitative case study of Middle East Respiratory Syndrome (MERS) and poliomyelitis in five member states. BMC Health Serv Res. 2018;18(1):528.

10. Bell DM, Weisfuse IB, Hernandez-Avila M, Del Rio C, Bustamante $\mathrm{X}$, Rodier G. Pandemic influenza as 21 st century urban public health crisis. Emerg Infect Dis. 2009;15(12):1963.

11. Rivera JD, Ceesay AA, Sillah A. Challenges to disaster risk management in The Gambia: A preliminary investigation of the disaster management system's structure. Prog Disaster Sci. 2020;6(3):100075.

12. Djalante R, Shaw R, DeWit A. Building resilience against biological hazards and pandemics: COVID-19 and its implications for the Sendai Framework. Prog Disaster Sci. 2020;100080.

13. Lak A, Hasankhan F, Garakani SA. Principles in practice : Toward a conceptual framework for resilient urban design. J Environ Plan Manag. 2020;1(1):1-33.

14. Vaništa Lazarević E, Keković Z, Antonić B. In search of the principles of resilient urban design: Implementability of the principles in the case of the cities in Serbia. Energy Build [Internet]. 2018 Jan [cited 2019 Jan 2];158:1130-8. Available from: https://www.sciencedirect.com/science/article/pii/S037877881730881 2.

15. Fekete A, Hufschmidt G, Kruse S. Benefits and challenges of resilience and vulnerability for disaster risk management. Int J disaster Risk Sci. 2014;5(1):3-20.

16. Brizuela NG, Garcia-Chan N, Pulido HG, Chowell G. Understanding the role of urban design in disease spreading. BioRxiv. 2019;766667.

17. Lee VJ, Aguilera X, Heymann D, Wilder-Smith A, Lee VJ, Heymann DL, et al. Preparedness for emerging epidemic threats: a Lancet Infectious Diseases Commission. Lancet Infect Dis. 2020;20(1):17-9.

18. Lee VJ, Ho M, Kai CW, Aguilera X, Heymann D, Wilder-Smith A. Epidemic preparedness in urban settings: new challenges and opportunities. Lancet Infect Dis. 2020; 20(5):527-9.

19. Garakani SA, Lak A, Niyasati M. Toward sustainable development in post-flood relocation of rural settlements in Iran. Int J Disaster Resil Built Environ [Internet]. 2020 Jan 31;11(3):359-77. Available from: https://www.emerald.com/insight/content/doi/10.1108/IJDRBE-05-20 19-0020/full/html

20. Golkar K. Creating sustainable place: reflections on urban design theory. Uni Shahid Beheshti Publ. 2011:112-46.

21. Neiderud CJ. How urbanization affects the epidemiology of emerging infectious diseases. Infect Ecol Epidemiol. 2015;5(1):27060.

22. Chandra S, Kassens-Noor E, Kuljanin G, Vertalka J. A geographic analysis of population density thresholds in the influenza pandemic of 1918-19. Int J Health Geogr. 2013;12(1):9.

23. Lin S, Qiao Y, Huang J, Yan N. Research on the Influence of Effective Distance Between Cities on the Cross-regional Transmission of COVID-19. medRxiv. 2020;1(1):1-22.

24. Hackl J, Dubernet T. Epidemic spreading in urban areas using agentbased transportation models. Futur Int. 2019;11(4):92.

25 Elrahman S, Meyer M. Transportation and Public Health: An Integrated Approach to Policy, Planning, and Implementation. Elsevier; 2019.

26. Allam Z, Jones DS. On the coronavirus (COVID-19) outbreak and the smart city network: universal data sharing standards coupled with artificial intelligence (AI) to benefit urban health monitoring and management. In: Healthcare. 2020. p. 46.

27. Sharifi A. Resilient urban forms: A macro-scale analysis. Cities. 2019;85(August 2018):1-14

28. World Health Organization. Infection prevention and control of epidemic-and pandemic-prone acute respiratory infections in health care. World Health Organization; 2014.

29. Coccia M. Two mechanisms for accelerated diffusion of COVID-19 outbreaks in regions with high intensity of population and polluting industrialization: the air pollution-to-human and human-to-human transmission dynamics. medRxiv. 2020.

30. Beelen R, Raaschou-Nielsen O, Stafoggia M, Andersen ZJ, Weinmayr G, Hoffmann B, et al. Effects of long-term exposure to air pollution on natural-cause mortality: an analysis of 22 European cohorts within the multicentre ESCAPE project. Lancet. 2014;383(9919):785-95.

31. Nenna R, Evangelisti M, Frassanito A, Scagnolari C, Pierangeli A, Antonelli G, et al. Respiratory syncytial virus bronchiolitis, weather conditions and air pollution in an Italian urban area: An observational study. Environ Res. 2017;158:188-93.

32. Liao CM, Hsieh NH, Chio CP. Fluctuation analysis-based risk assessment for respiratory virus activity and air pollution associated 
asthma incidence. Sci Total Environ. 2011;409(18):3325-33.

33. Glencross DA, Ho TR, Camiña N, Hawrylowicz CM, Pfeffer PE. Air pollution and its effects on the immune system. Free Radic Biol Med. 2020;151(1): 56-68.

34. Tambo E, Ngogang JY, Ning X, Xiao-Nong Z. Strengthening community support, resilience programmes and inter-ventions in infectious diseases of poverty. East Mediterr Health J. 2018;24(62018).

35. Tang L, Bie B, Park SE, Zhi D. Social media and outbreaks of emerging infectious diseases: A systematic review of literature. Am J Infect Control. 2018;46(9):962-72.

36. Tay J, Ng YF, Cutter J, James L. Influenza A (H1N1-2009) pandemic in Singapore-public health control measures implemented and lessons learnt. Ann Acad Med Singapore. 2010;39(4):313.

37. Ahn I, Heo S, Ji S, Kim KH, Kim T, Lee EJ, et al. Investigation of nonlinear epidemiological models for analyzing and controlling the MERS outbreak in Korea. J Theor Biol. 2018;437:17-28.

38. Stier A, Berman M, Bettencourt L. COVID-19 attack rate increases with city size. Mansueto Inst Urban Innov Res Pap Forthcom. 2020; Available at SSRN: https://ssrn.com/abstract=3564464

39. World Health Organization. Considerations for quarantine of individuals in the context of containment for coronavirus disease (COVID-19): interim guidance, 19 March 2020. 2020. 